as a means of control of noise-induced motion // Phys. Rev. Lett. 2004. Vol. 93010601.

18. Prager T., Lerch H.-Ph., Schimansky-Geier L., Schöll E. Increase of coherence in excitable systems by delayed feedback // J. Phys. A : Math. Theor. 2007. Vol. 40. P. 11045-11055.

19. Brandstetter S., Dahlem M. A., Schöll E. Interplay of time-delayed feedback control and temporally correlated noise in excitable systems // Philosophical Transactions of the Royal Society A: Mathematical, Physical and Engineering Sciences. 2010. Vol. 368. P. 391-421.

20. Geffert P. M., Zakharova A., Vüllings A., Just W., Schöll E. Modulating coherence resonance in nonexcitable systems by time-delayed feedback // Eur. Phys. J. B. 2014. Vol. 87. P. 291-304.
21. Escalera Santos G. J., Escalona J., Parmananda P. Regulating noise-induced spiking using feedback // Phys. Rev. E. 2006. Vol. 73. P. 042102.

22. Semenov V., Feoktistov A., Vadivasova T., Schöoll E., Zakharova A. Time-delayed feedback control of coherence resonance near subcritical Hopf bifurcation : Theory versus experiment// Chaos. 2015. Vol. 25. P. 033111.

23. FitzIIugh R. Impulses and physiological states in theoretical models of nerve membrane // Biophysical J. 1961. Vol. 1 (6). P. 445-466.

24. Nagumo J. S., Arimoto S., Yoshizawa S. An active pulse transmission line simulating nerve axon // Proceedings of the Institute of Radio Engineers. 1962. Vol. 50. P. 2061-2071.

\title{
ПОЛНОЕ ПРЕОБРАЗОВАНИЕ ПОЛЯРИЗАЦИИ ТЕРАГЕРЦОВОГО ИЗЛУЧЕНИЯ МАССИВОМ ГРАФЕНОВЫХ МИКРОЛЕНТ БЕЗ ИСПОЛЬЗОВАНИЯ МАГНИТНОГО ПОЛЯ
}

\author{
В. С. Мельникова ${ }^{1}$, О. В. Полищук², В. В. Попов ${ }^{1,2}$ \\ ${ }^{1}$ Саратовский государственный университет \\ ${ }^{2}$ Саратовский филиал Института радиотехники \\ и электроники им. В. А. Котельникова РАН \\ E-mail: MelnikovaVeronica@yandex.ru
}

\begin{abstract}
Исследовано преобразование поляризации терагерцового излучения периодическим массивом графеновых микролент, расположенных на поверхности диэлектрика (призмы) с высоким показателем преломления. Преобразование поляризации на частоте плазменного резонанса происходит без приложения внешнего постоянного магнитного поля. Показано, что гигантское преобразование поляризации может быть достигнуто при полном внутреннем отражении терагерцовой волны от периодического массива графеновых микролент.
\end{abstract}

Ключевые слова: терагерцовое излучение, графен, плазмоны, преобразование поляризации.

Total Conversion of Terahertz Wave Polarization by Graphene Microribbon Array without Magnetic Field

\section{S. Melnikova, O. V. Polischuk, V. V. Popov}

The polarization conversion of terahertz radiation by the periodic array of graphene microribbons located at the surface of a high-refractiveindex dielectric substrate (prizm) is studied theoretically. Polarization conversion at the plasmon resonance frequencies takes place without applying external DC magnetic field. It is shown that giant (up to total) polarization conversion can be reached at the total internal reflection of THz wave from the periodic array of graphene nanoribbons.

Key words: terahertz radiation, graphene, plasmons, polarization conversion.

DOI: 10.18500/1817-3020-2015-15-3-51-54

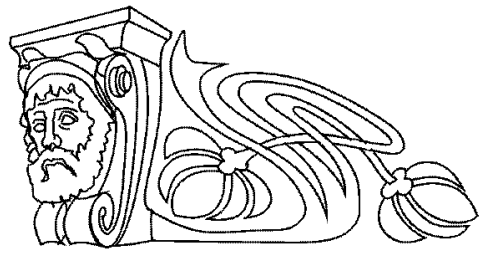

\section{Введение}

Известно, что преобразование поляризации электромагнитной волны может иметь место исключительно в системах, не обладающих зеркальной плоскостью симметрии. Обычно для нарушения зеркальной симметрии используют внешнее постоянное магнитное поле [1]. В практическом плане это приводит к существенному росту габаритов и веса преобразователей поляризации.

Графен демонстрирует сильный плазмонный отклик на терагецовых (ТГц) частотах, что обусловлено как высокой плотностью, так и малой коллективной динамической массой свободных носителей $[2,3]$.

В данной работе теоретически исследуется прсобразование поляризации ТГц волны двумерным периодическим массивом графеновых микролент, расположенных на поверхности раздела диэлектрика (призмы) с высоким показателем преломления. Показано, что в данной структуре возможно гигантское (практически полное) преобразование поляризации ТГц волны в режиме полного внутреннего отражения волны 
от массива графеновых микролент в отсутствие внешнего постоянного магнитного поля.

\section{1. Теоретическая модель}

Периодический массив графеновых микролент находится на поверхности диэлектрика (призмы) с высокой диэлектрической постоянной $\varepsilon_{2}=11,45$ (Si) (рис. 1). Плоская р-поляризованная ТГц волна падаст на графен под углом $\theta$. Направлснис периодичности массива графеновых микролснт образует угол ф по отношению к плоскости падсния ТГц волны. Нарушение зеркальной симметрии структуры, необходимое для преобразования поляризации, достигается при наклонном падении ТГц волны, когда направление периодичности массива графеновых микролент не лежит в плоскости падения падающей ТГц волны $(\varphi \neq 0)$.

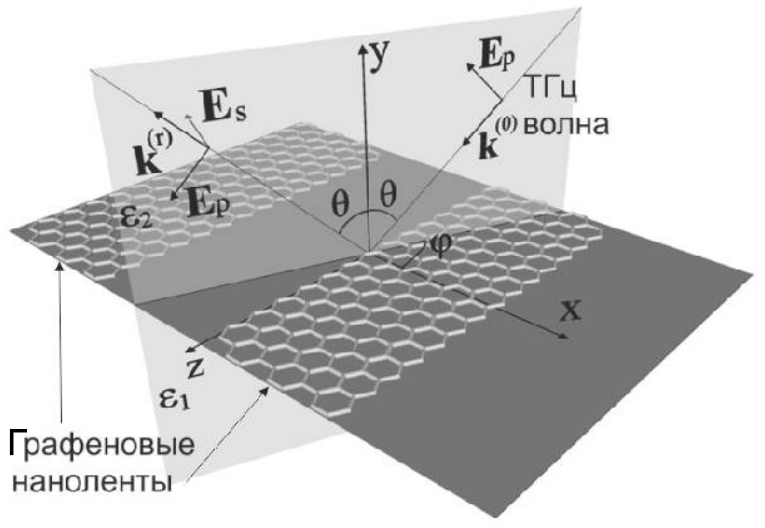

Рис. 1. Схематическое изображение структуры. Внешняя $p$-поляризованная ТГц волна падает на массив графеновых микролент под углом $\theta$ изнутри более оптически плотной среды. Направление периодичности графеновых микролент образует угол $\varphi$ по отношению к плоскости падения $p$-поляризованной ТГц волны

Решается полная система уравнений Максвелла с разложснисм искомых элсктричсских и магнитных полей по плоским волнам. Вследствие периодичности структуры в $x$-направлении индуцированные электрические и магнитные поля можно разложить в ряд Фурье и переписать уравнения Максвелла в каждой среде в фурьепредставлении. Использование стандартных электромагнитных граничных условий в плоскости $y=0$ (в плоскости массива графеновых микролент) позволяет получить соотношение между амплитудами фурье-гармоник плотности элсктричсского тока и элсктричсского поля в этой плоскости. Графен описывается с помощью динамической проводимости [4]:

$$
\begin{aligned}
& \frac{\sigma_{G r}(\omega)}{\sigma_{0}}=\frac{8 i k_{B} T}{\pi \hbar(\omega+\mathrm{i} \gamma)} \ln \left[2 \cosh \left(\frac{E_{F}}{2 k_{B} T}\right)\right]+ \\
& +G\left(\frac{\hbar \omega}{2}\right)+\frac{4 i \hbar \omega}{\pi} \int_{0}^{\infty} \frac{G(\varsigma)-G(\hbar \omega / 2)}{(\hbar \omega)^{2}-4 \varsigma^{2}} d \varsigma,
\end{aligned}
$$

где

$$
G(\varsigma)=\frac{\sinh \left(\varsigma / k_{B} T\right)}{\cosh \left(E_{F} / k_{B} T\right)+\cosh \left(\varsigma / k_{B} T\right)} .
$$

Здесь $\sigma_{0}=e^{2} / 4 \hbar, E_{F}$ - энергия Ферми, температура $T$ полагается равной $300 \mathrm{~K}, \omega-$ круговая частота падающей ТГ ц электромагнитной волны, $e$ - заряд э.тектрона, $k_{B}-$ постоянная Больцмана и $\hbar$ - приведенная постоянная Планка. Первое слагаемое в формуле (1) описывает отклик в форме Друде с участием внутризонных процессов, описываемых феноменологической скоростью внутризонного расссяния элсктронов $\gamma$. Второе слагаемое описывает межзонные персходы в графене.

Используя закон Ома в графене с проводимостью (1) совместно с выражением для плотности электрического тока, полученного из электромагнитных граничных условий, находим систему связанных интегральных уравнений для $x$ - и $z$-компонент плотности электрического тока в графеновых микролентах. Данная система связанных интегральных уравнений решалась численно методом Галеркина с разложением искомых функций по ортогональным полиномам Лежандра.

\section{2. Результаты и их обсуждение}

На рис. 2 показан спектр ТГц поглощения в зависимости от величины энергии Ферми и частоты для режима полного внутреннего отражения (ПВО) $\theta>\theta_{\mathrm{R}}$, где $\theta_{\mathrm{R}}-$ угол полного внутреннего отражения $\left(\theta_{\mathrm{R}} \approx 17^{\circ}\right.$ при $\left.\varepsilon_{2}=11,45\right)$ при $\gamma=0$. В этом случас поглошснис ТГц волны вызвано исключительно межзонной генерацией элсктронно-дырочных пар в графене и умсньшастся с увсличснисм энсргии Ферми. Светлые области на рис. 2 соответствуют основному и высшему плазмонным резонансам.

Тонкие светлые линии соответствуют слабому ТГц поглощснию на частотах сателлитов основного и высшего плазмонных резонансов. Эти сателлиты не проявляются в спектрах поглощсния в симметричной системе (при $\varphi=0$ ) и приводят к слабому резонансному поглощению только при отсутствии зеркальной симметрии в рассматриваемой системе при $\varphi \neq 0$ и $0 \neq 0$. 


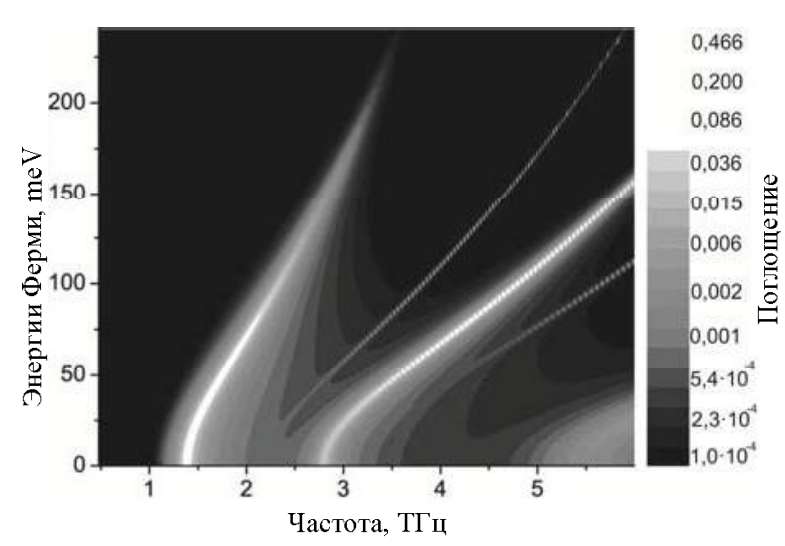

Рис. 2. Спектр ТГц поглощения в режиме полного внутреннего отражения $\left(\theta=\varphi=45^{\circ}\right)$ для массива графеновых микролент шириной 1 мкм с периодом 2 мкм как функция величины энергии Ферми и частоты для $\gamma=0$. Светлые области соответствуют основному и высшему плазмонным резонансам

Определим коэффициент преобразования поляризации как отношение мсжду потоками мощности $s$-поляризованной отраженной волны и падающей $p$-поляризованной волны. Как слсдуст из рис. 3 , коэффициент преобразования поляризации возрастает на несколько порядков величины на частотах плазменных резонансов и достигает единицы (что соответствует полному преобразованию поляризации) при высоких значениях энергии Ферми для случая $\gamma=0$. Коэффициент преобразования поляризации и ширина резонанса преобразования поляризации возрастают вдоль лепестка плазмонного резонанса с ростом энергии Ферми вслсдствие того, что межзонное поглощение умсньшастся с увсличснисм энсргии Ферми.

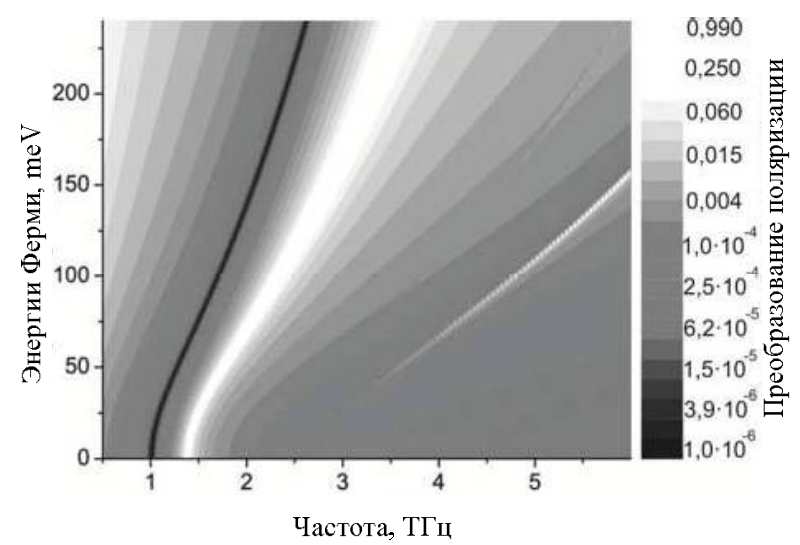

Рис. 3. Коэффициент преобразования поляризации в основном плазмонном резонансе в рсжиме полного внутреннего отражения $\left(\theta=\varphi=45^{\circ}\right)$ для массива графеновых микролент шириной 1 мки с периодом 2 мкм как функция энергии Ферми и частоты при $\gamma=0$
Нулевые значения преобразования поляризации (черная линия на рис. 3) в низкочастотной области фундаментального плазмонного резонанса возникает из-за асимметричной (типа Фано) формы резонанса преобразования поляризации.

Поскольку период рассматривасмого массива графеновых микролснт намного меньше, чем длина волны падающей ТГц волны, массив можно рассматривать как анизотропную резонансную метаповерхность. Преобразование поляризации ТГц волны возникает из-за анизотропии плазмонного отклика массива графеновых микролент для компонент электрического поля, направленного вдоль и поперек графеновых микролент. Полное преобразование поляризации возникает в том случае, когда отраженная волна с исходной поляризацией полностью гасится отраженной волной с той же поляризацией, индуцированной плазменными колебаниями в массиве графеновых микролснт.

Расчеты, выполненные для рсалистичных параметров массива графеновых микролент при комнатной температуре (рис. 4), показывают возможность получения гигантских (до $70 \%$ ) значений коэффициента преобразования поляризации ТГц волны по отношению к падающей волне. Часть мощности (порядка $30 \%$ ) падающей волны теряется за счет поглощения в графене, главным образом, в результате внутризонных процессов рассеяния свободных носителей заряда (первос слагаемое в формуле (1) при $\gamma=0$. В то же врсмя отражснная от массива графеновых микролснт ТГц волна имеет строго ортогональную поляризацию элсктрического поля по отношению к падающсй волне.

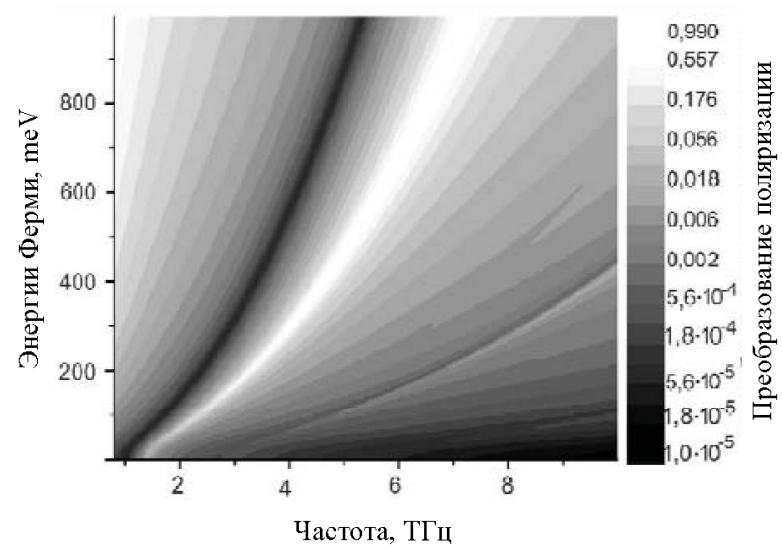

Рис. 4. Коэффициент преобразования поляризации в основном плазионном резонансе в режиме полного внутреннего отражения $\left(\theta=\varphi=45^{\circ}\right)$ для массива графеновых микролент шириной 1 мкм с периодом 2 мки как функция энергии Ферми и частоты при $\gamma=10^{12} \mathrm{c}^{-1}$ 
Заметим, что процесс преобразования поляризации волны в данной структуре является взаимным. Таким образом, падающая ТГц волна с $s$-поляризацией будет преобразовываться в волну с $p$-поляризацией с тем же самым коэффициентом преобразования.

Работа выполнена при финансовой поддержке РФФИ (проект io 14-02-92102).

\section{Список литературы}

1. Crassee I., Levallois J., Walter A. L., Ostler M., Bostwick A., Rotenberg E., Seyller T., D., van der Marell, Kuzmenko A. B. Giant Faraday rotation in single- and multilayer graphene // Nature Physics. 2011. Vol. 7. P. $48-51$.

2. Chen J., Badioli M., Alonso-González P., Thongrattanasiri S., Huth F., Hillenbrand R., Koppens F. Optical nano-imaging of gate-tunable graphene plasmons // Nature. 2012. Vol. 487. P. 77-81.

3. Fei Z., Rodin A. S., Andreev G. O., Bao W., McLeod A. S., Wagner M., Zhang L. M., Zhao Z., Thiemens M., Dominguez G., Fogler M. M., Castro Neto A. II., Lau C. N., Keilmann F, Basov D. N. Gate-tuning of graphene plasmons revealed by infrared nano-imaging // Nature. 2012. Vol. 487. P. 82-85.

4. Falkovsky L. A., Varlamov A. A. Space-time dispersion of graphene conductivity // Eur. Phys. J. B. 2007. Vol. 56, № 4. P. 281-284. 